\title{
Ligand-Mediated Band Engineering in Bottom-Up Assembled SnTe Nanocomposites for Thermoelectric Energy Conversion
}

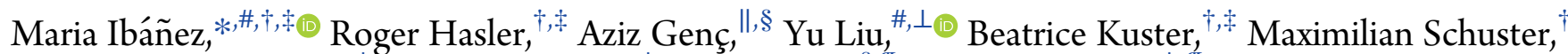

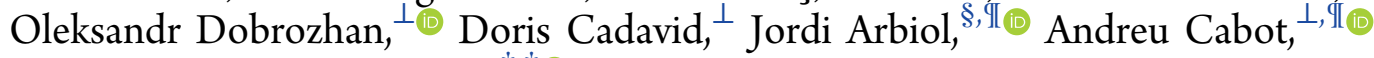 \\ and Maksym V. Kovalenko*,†,\$(0) \\ \#Institute of Science and Technology Austria, Am Campus 1, 3400 Klosterneuburg, Austria \\ ${ }^{\dagger}$ Institute of Inorganic Chemistry, Department of Chemistry and Applied Biosciences, ETH Zürich, Vladimir Prelog Weg 1, Zürich \\ CH-8093, Switzerland \\ ${ }^{\ddagger}$ Empa-Swiss Federal Laboratories for Materials Science and Technology, , Überlandstrasse 129, Dübendorf CH-8600, Switzerland \\ "Department of Metallurgy and Materials Engineering, Faculty of Engineering, Bartin University, 74100 Bartin, Turkey \\ ${ }^{\S}$ Catalan Institute of Nanoscience and Nanotechnology (ICN2), CSIC and BIST, Campus UAB, Bellaterra, 08193 Barcelona, \\ Catalonia, Spain \\ ${ }^{\perp}$ Catalonia Energy Research Institute-IREC, Sant Adrià del Besòs, 08930 Barcelona, Spain \\ IICREA, Pg. Lluís Companys 23, 08010 Barcelona, Spain
}

Supporting Information

\begin{abstract}
The bottom-up assembly of colloidal nanocrystals is a versatile methodology to produce composite nanomaterials with precisely tuned electronic properties. Beyond the synthetic control over crystal domain size, shape, crystal phase, and composition, solution-processed nanocrystals allow exquisite surface engineering. This provides additional means to modulate the nanomaterial characteristics and particularly its electronic transport properties. For instance, inorganic surface ligands can be used to tune the type and concentration of majority carriers or to modify the electronic band structure. Herein, we report the thermoelectric properties of $\mathrm{SnTe}$ nanocomposites obtained from the consolidation of surface-engineered SnTe nanocrystals into macroscopic pellets. A CdSe-based ligand is selected to (i) converge the light and heavy bands through partial $\mathrm{Cd}$ alloying and (ii) generate CdSe nanoinclusions as a secondary phase within the SnTe matrix, thereby reducing the thermal conductivity. These SnTe-CdSe nanocomposites possess thermoelectric figures of merit of up to 1.3 at $850 \mathrm{~K}$, which is, to the best of our knowledge, the highest thermoelectric figure of merit reported for solutionprocessed SnTe.
\end{abstract}

$\mathrm{T}$ he search for thermoelectric materials, able to efficiently convert heat into electricity (and vice versa), has been a longstanding challenge. A material's thermoelectric performance is usually evaluated through a dimensionless figure of merit, $Z T$, defined as $Z T=\sigma S^{2} T \kappa^{-1}$ where $T$ is the absolute temperature, $\sigma$ the electrical conductivity, $S$ the Seebeck coefficient or thermopower, and $\kappa$ the thermal conductivity. ${ }^{1}$ All these parameters are governed by the electronic structure of the material and its charge and phonon scattering properties. ${ }^{2}$ Several of the current best-performing thermoelectric materials are nanocomposites produced via spinodal decomposition during solid-state synthesis, resulting in a crystalline matrix containing nanoinclusions of a second phase. ${ }^{3}$ Alternatively, the bottom-up assembly of colloidally synthesized, solutionprocessed nanocrystals (NCs) has recently emerged as a convenient methodology to produce high-ZT materials. ${ }^{4,5}$ Using this strategy, materials such as $\mathrm{Cu}_{3} \mathrm{Sb}_{1-x-y} \mathrm{Sn}_{x} \mathrm{Bi}_{y} \mathrm{Se}_{4}{ }^{6}$ $\left(\mathrm{Ag}_{2} \mathrm{Te}\right)_{5}\left(\mathrm{Sb}_{2} \mathrm{Te}_{3}\right)_{5},{ }^{7} \quad \mathrm{AgBi}_{0.5} \mathrm{Sb}_{0.5} \mathrm{Se}_{2}{ }^{8} \mathrm{PbTe} \mathrm{Bi}_{0.7} \mathrm{Sb}_{1.3} \mathrm{Te}_{3},{ }^{\prime}$, $\mathrm{Bi}_{2} \mathrm{Te}_{2.7} \mathrm{Se}_{0.3}$, ${ }^{10}$ and $\mathrm{Bi}_{0.5} \mathrm{Sb}_{1.5} \mathrm{Te}_{3}{ }^{11}$ have reached $\mathrm{ZT}$ above 1 . Despite these promising results, further progress requires precise surface engineering of the NCs and methods to control the NC assembly and consolidation into bulk nanocrystalline materials. Most colloidal syntheses deliver NCs with organic molecules (ligands) coordinated at their surfaces. ${ }^{12}$ These molecules are highly insulating and thus need to be removed prior to NCs consolidation. Usually, such organic ligands are thermally decomposed, chemically displaced, or exchanged with inorganic ligands to produce fully inorganic nanomaterials. However, beyond avoiding detrimental effects of bulky organic ligands, surface modification can be envisioned as a powerful tool to further adjust the final nanocomposite composition and therefore its functional properties. Recent work has demonstrated the possibility to tune the type and concentration of majority carriers with aliovalent electronic impurities, incorporated through surface ligands. ${ }^{13,14}$ In addition, ligands have been proposed as molecular solders to facilitate charge transport between the grain boundaries. ${ }^{15,16}$

Here, we demonstrate that ligands can be also used to modify the electronic band structure of the final nanocomposite, while simultaneously acting as precursors for the formation of secondary phases during NCs consolidation.

In particular, we focus on the development of SnTe-based nanocomposites from SnTe NCs (Figure 1). While very few

Received: February 5, 2019

Published: April 19, 2019 


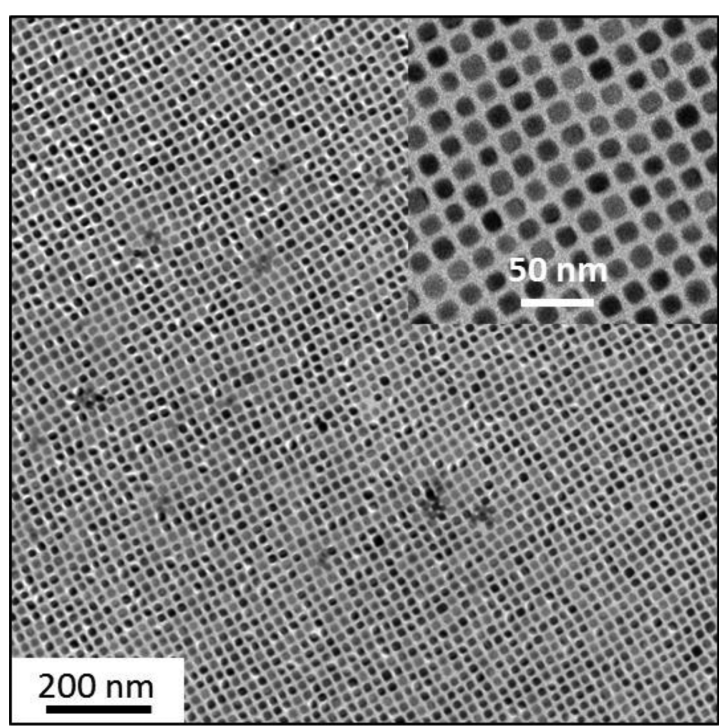

Figure 1. TEM images of the as-prepared SnTe NCs.

reports have centered on the synthesis and assembly of SnTe NCs, ${ }^{17-19}$ the highest $Z T$ value (ca. 0.5 at $800 \mathrm{~K}$ ) obtained from bottom-up assembled $\mathrm{SnTe}$ was reached using rather large (ca. $170 \mathrm{~nm}$ ) particles consolidated via spark plasma sintering. Such nanomaterials showed lower lattice thermal conductivities, 0.52 $\mathrm{W} \mathrm{m}{ }^{-1} \mathrm{~K}^{-1}$, than bulk SnTe, $1.6 \mathrm{~W} \mathrm{~m}^{-1} \mathrm{~K}^{-1}$. ${ }^{20}$ However, they featured lower electrical conductivities $\left(500 \mathrm{~S} \mathrm{~cm}^{-1}\right.$ for NC based solid $^{18}$ compared to $1000 \mathrm{~S} \mathrm{~cm}^{-1}$ for bulk $\mathrm{SnTe}^{20}$ ) and lower Seebeck coefficients $\left(90 \mu \mathrm{V} \mathrm{K}^{-1}\right.$ versus $\left.130 \mu \mathrm{V} \mathrm{K} \mathrm{K}^{-1}\right)$. Consequently, the power factor $\left(P F=\sigma S^{2}\right)$ was approximately 4 times smaller than that of bare $\mathrm{SnTe}$ solids. In order to overcome such limitations, we explored the use of SnTe NCs as building blocks to produce SnTe-based nanomaterials.

SnTe NCs were prepared by reacting tin bromide with trioctylphosphine telluride in the presence of oleylamine and trioctylamine (experimental details can be found in the Supporting Information (SI)). The obtained NCs were purified inside an argon-filled glovebox to prevent surface oxidation. Figure 1 shows representative transmission electron microscopy (TEM) images of the obtained SnTe NCs, exhibiting cubic morphology and an average side length of $14 \pm 1 \mathrm{~nm}$.

In order to evaluate thermoelectric properties of SnTe-based nanomaterials obtained from the assembly of these NCs, we first displaced native organic ligands from the NC surface. For this purpose, we mixed an ammonium thiocyanate $\left(\mathrm{NH}_{4} \mathrm{SCN}\right)$ solution in anhydrous methanol with $\mathrm{SnTe}$ NCs suspended in anhydrous tetrahydrofuran (see SI). $\mathrm{NH}_{4} \mathrm{SCN}$ efficiently replaced organic ligands ${ }^{21,22}$ and allowed the production of fully inorganic $\mathrm{SnTe}$ nanosolids (further referred to as $\mathrm{SnTe}$ SCN) through hot pressing of the organic-free NCs. Details of the NC consolidation can be found in the SI. Figure 2 displays the thermoelectric properties of SnTe-SCN nanomaterial. Hall charge carrier concentrations of $2 \times 10^{20} \mathrm{~cm}^{-3}$ and electrical conductivities up to $720 \mathrm{~S} \mathrm{~cm}^{-1}$ were measured at room temperature. However, low Seebeck coefficients (ca. $110 \mu \mathrm{V}$ $\mathrm{K}^{-1}$ ) and high thermal conductivities (ca. $2.25 \mathrm{~W} \mathrm{~m}^{-1} \mathrm{~K}^{-1}$ ) provided maximum $Z T$ values of just 0.35 at $850 \mathrm{~K}$.

SnTe has a high symmetry cubic crystal structure and a complex valence band structure with a small band gap $(0.18 \mathrm{eV}$ at room temperature), which favors bipolar charge transport at relatively low temperatures. ${ }^{23}$ It is also characterized by a large a)
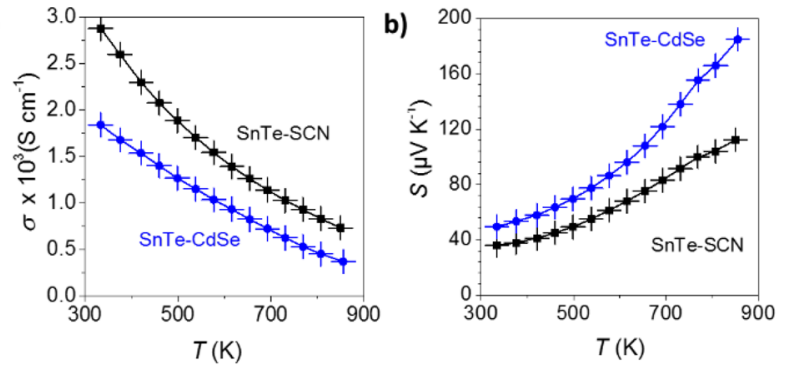

c)

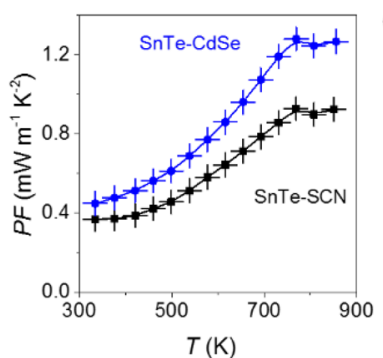

d)

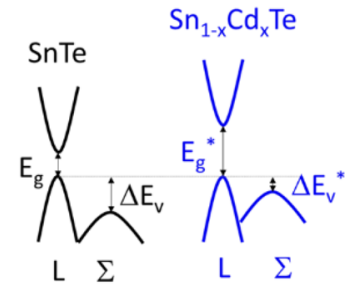

e)
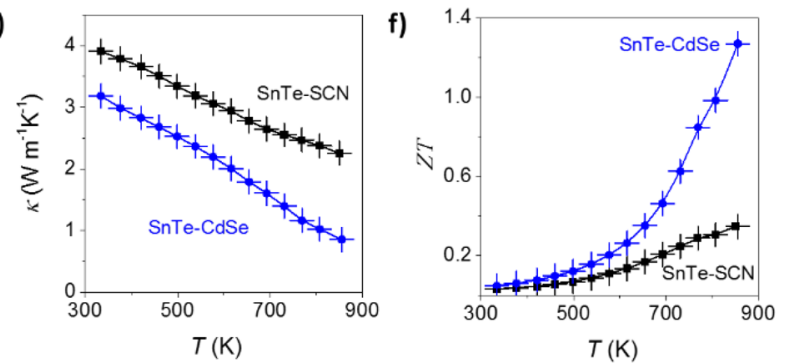

Figure 2. (a) Electrical conductivity, $\sigma$; (b) Seebeck coefficient, $S$; (c) power factor, $P F$; (d) schematic of the electronic band structure; (e) thermal conductivity, $\kappa$; and (f) figure of merit, $Z T$, for $S n T e$ nanomaterial prepared with SCN (black) and CdSe (blue) surfacemodified SnTe NPs.

offset $(0.3-0.4 \mathrm{eV})^{24,25}$ between the light valence band $(\mathrm{L})$ and the heavy valence band $(\Sigma)$ that prevents the contribution of the heavier holes to the Seebeck coefficient. ${ }^{26}$ Additionally, the offstoichiometric nature of $\mathrm{SnTe}$ with a large number of $\mathrm{Sn}$ lattice vacancies yields very high intrinsic doping levels $\left(p=10^{21}\right.$ $\left.\mathrm{cm}^{-3}\right) .^{27}$ These characteristics are far from the optimum for thermoelectric applications, what translates into low Seebeck coefficients and high thermal conductivities, and makes SnTe a poor thermoelectric material. ${ }^{26}$ Overall, the success toward producing SnTe with high $\mathrm{ZT}$ should rely on the carrier concentration optimization, a proper band structure engineering, and the introduction of all-scale (atomic, nano, and meso) hierarchical scattering centers. ${ }^{28}$

Previous reports show that the hole carrier concentration could be reduced by introducing donors such as $\mathrm{I}^{29} \mathrm{Bi}^{30}$ or $\mathrm{Sb},{ }^{30}$ as well as by $\mathrm{Sn}$ self-compensation $\left(\mathrm{Sn}_{1+x} \mathrm{Te}\right) \cdot{ }^{31,32}$ However, the band structure could be modified to introduce resonant states in the valence band near the Fermi level via In doping ${ }^{20}$ or to promote band convergence and open the band gap via $\mathrm{Cd}^{20,31,33-35} \mathrm{Hg}^{36} \mathrm{Mg},{ }^{37} \mathrm{Ca},{ }^{38}$ and $\mathrm{Mn}^{39,40}$ alloying. Both strategies allowed for higher Seebeck coefficients. Further strategies to reduce the lattice thermal conductivity are based on the introduction of nanoscale secondary phases such as $\mathrm{SrTe}^{30}{ }^{30} \mathrm{AgBiTe}_{2},{ }^{41} \mathrm{AgInTe}_{2},{ }^{42} \mathrm{In}_{2} \mathrm{Te}_{3},{ }^{43} \mathrm{GaTe},{ }^{44} \mathrm{CdS},{ }^{31}$ and $\mathrm{ZnS}^{31}$ within the $\mathrm{SnTe}$ matrix via solid state precipitation.

Inspired by the enhancement of the Seebeck coefficient achieved in SnTe through band convergence and widening of the 

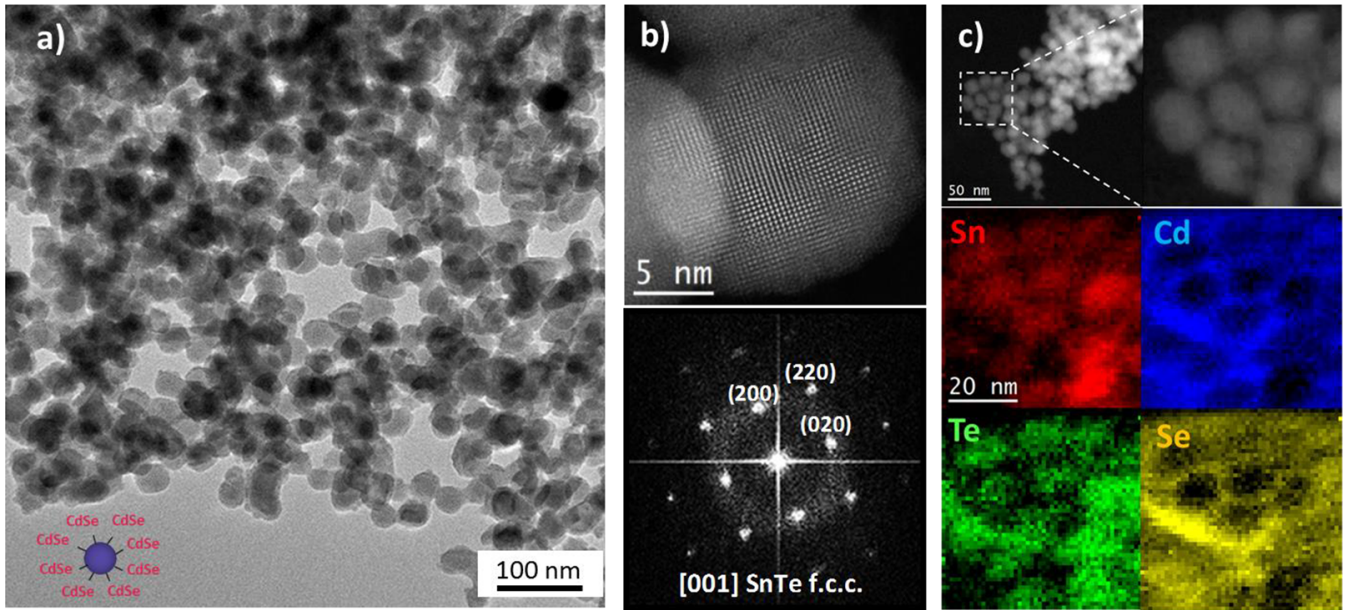

Figure 3. (a) General TEM micrograph of the SnTe@CdSe core-shell like nanoparticles, revealing the homogeneity in size and shape. (b) Atomic resolution HAADF STEM micrograph of a core-shell nanoparticle and its corresponding power spectrum. (c) ADF STEM micrograph of several SnTe@CdSe NPs and the corresponding STEM-EELS elemental composition maps: Sn (red), Te (green), Cd (blue), and Se (yellow).

band gap via alloying with transition metals, and specifically with $\mathrm{Cd}{ }^{20,31,33,34}$ we designed a ligand exchange strategy using soluble CdSe-compounds as inorganic ligands. The CdSe-based ligand was prepared by dissolving stoichiometric amounts of elemental $\mathrm{Cd}$ and $\mathrm{Se}$ in a thiol-amine mixture ${ }^{45}$ at room temperature and in inert-atmosphere (SI). This molecular solution (referred to as CdSe ink) allowed the recovery of pure hexagonal $\mathrm{CdSe}$ upon mild annealing at $350{ }^{\circ} \mathrm{C}$ (Figure S1). We hypothesize that the ligand could be a variety of chalcogenidocadmates such as $\left(\mathrm{Cd}_{2} \mathrm{Se}_{3}\right)_{n}{ }^{2 n-}$ or $\mathrm{CdSe}_{2}{ }^{2-}$ as previously reported for hydrazinum based CdSe solutions. ${ }^{46,47}$ In order to replace the organic ligands on the NC surface by the CdSe-based ligand, a phase transfer reaction was used: a solution of SnTe NCs in hexane was layered on top of a solution of the CdSe ink in $N$ methylformamide. The change of colloidal stabilization from steric to electrostatic was evidenced by the color change of the solutions, reaching a colorless hexane phase and colored polar phase. The top hexane phase was then discarded and the SnTe NCs were precipitated by centrifugation, rinsed twice with acetone, and dried under vacuum for future use.

Figure 3a shows TEM images of CdSe-functionalized SnTe NCs (SnTe@CdSe).Atomic resolution high-angle annular darkfield (HAADF) scanning TEM (STEM), demonstrated that the NCs were composed of a $\sim 1 \mathrm{~nm}$ thick amorphous shell around a perfectly crystalline $\sim 14 \mathrm{~nm}$ core (Figure $3 \mathrm{~b}$ and SI). The power spectrum (FFT) obtained from these nanoparticles revealed their core to have a crystal structure matching an fcc SnTe phase visualized along its [001] zone axis. To unravel the composition of the amorphous shell, STEM electron energy loss spectroscopy (EELS) analyses were conducted. EELS compositional maps indicated the presence of amorphous CdSe shells at the surface of SnTe cores. More examples can be found in Figures S5 and S6.

Subsequently, SnTe@CdSe nanoparticles were used as building blocks to produce $\mathrm{SnTe}-\mathrm{CdSe}$ nanocomposites under the same conditions as SnTe-SCN nanomaterials. A $6.4 \%$ content of $\mathrm{Cd}$ was estimated considering the ligand surface coverage and verified by ICP (Table S1). Figure 2 displays the thermoelectric properties of these materials. Compared to SnTeSCN nanomaterials, the electrical conductivity of SnTe-CdSe nanocomposite was reduced from 720 to $380 \mathrm{~S} \mathrm{~cm}^{-1}$, while the Seebeck coefficient increased from 110 up to $185 \mu \mathrm{V} \mathrm{K}^{-1}$ at 850 $\mathrm{K}$. These results are in agreement with the predicted band structure modification of SnTe with the incorporation of $\mathrm{Cd}$ (Figure 2d). ${ }^{31}$ Substituting $\mathrm{Sn}$ atoms with isovalent $\mathrm{Cd}$ significantly increased the band gap and diminished the energy separation between the light-hole band and the heavy-hole band (Figure $2 \mathrm{~d}) .^{31}$ The larger band gap and the reduced carrier concentration $\left(9 \times 10^{19} \mathrm{~cm}^{-3}\right)$ of SnTe-CdSe also reduced bipolar contributions. Additionally, the convergence of the valence bands provided a higher hole density at the Fermi level. Both phenomena translated into much higher Seebeck coefficients in the whole temperature range, and especially at high temperatures. ${ }^{20,31}$ Thus, much higher power factors of ca. $1.3 \mathrm{~mW} \mathrm{~m}^{-1} \mathrm{~K}^{-2}$ were reached. Finally, thermal conductivities of $0.85 \mathrm{~W} \mathrm{~m}^{-1} \mathrm{~K}^{-1}$ were systematically acquired at $850 \mathrm{~K}$, which is approximately 2.7 times lower than for SnTe-SCN, and corresponded to a lattice thermal conductivities of $0.48 \mathrm{~W} \mathrm{~m}^{-1}$ $\mathrm{K}^{-1}(\mathrm{SI})$, close to the theoretical amorphous limit $\left(0.5 \mathrm{~W} \mathrm{~m}^{-1}\right.$ $\left.\mathrm{K}^{-1}\right) .{ }^{36}$

To understand the observed low lattice thermal conductivities, the mesoscale structure of $\mathrm{SnTe}-\mathrm{CdSe}$ nanocomposites was investigated (Figures 4 and S7-S9). HRTEM analysis showed the presence of $\mathrm{CdSe}$ precipitates, both with cubic or hexagonal phases, and with sizes between 3 and $10 \mathrm{~nm}$, within the SnTe matrix. In some cases, both crystal domains, SnTe and CdSe, were found to be in perfect epitaxy due to their low lattice mismatch of $1.8 \%$ (Figure S8). Overall, the alloy formation, the presence of nanoscale $\mathrm{CdSe}$ secondary phases, and the mesoscale SnTe domains provided scattering mechanisms to block propagation of phonons on all relevant length scales, thereby reducing the lattice thermal conductivity to the amorphous limit.

In conclusion, a scalable high-yield colloidal synthetic route to prepare SnTe NCs with narrow size distribution was presented. In order to optimize the thermoelectric performance of bottomup SnTe-based nanosolids, a new NC ligand-exchange process using CdSe-based compounds was presented. Upon thermal consolidation, the surface-deposited CdSe units partially alloyed with SnTe to modify its band structure and partially crystallized in CdSe nanodomains within the SnTe matrix. Alloying resulted in an increase of the Seebeck coefficient due to the influence of $\mathrm{Cd}$ in the electronic band structure of SnTe. Besides, the mesoscale structuration allowed reducing the thermal conductivity to the amorphous limit. Overall, SnTe-CdSe nanocomposites with $Z T$ of ca. 1.3 at $850 \mathrm{~K}$ were produced. 
a)

\section{b)}
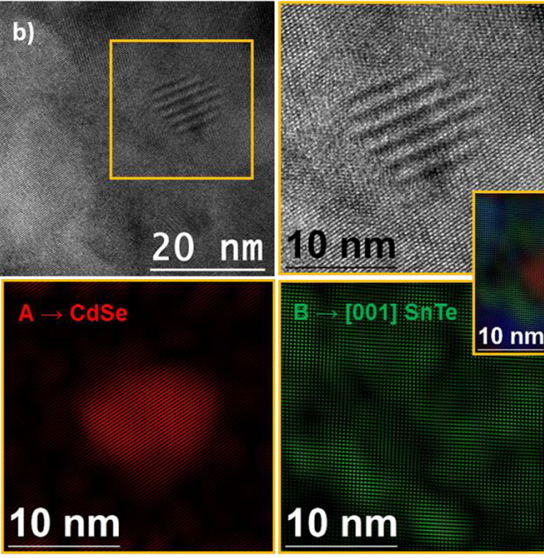

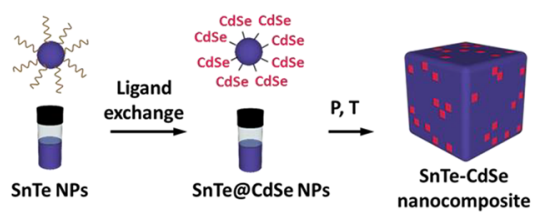

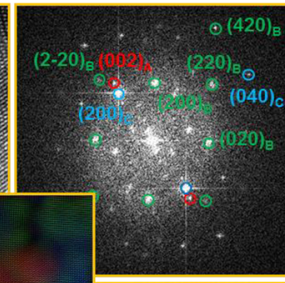

$10 \mathrm{~nm}$

$C \rightarrow[001]$ SnTe

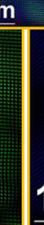

Figure 4. (a) Schematic of the bottom-up process. (b) HRTEM micrograph obtained from the cross-section of the SnTe-CdSe pellet sample with the corresponding power spectrum (FFT) from the marked yellow squared area. In the bottom line, phase filtered images correspond to a CdSe precipitate (red); a SnTe crystal domain is visualized along the [001] zone axis (green) and another SnTe crystal domain is also visualized along the [001] zone axis (blue). The inset below corresponds to a phase-filtered RGB composition map showing the different superposed phases found.

\section{ASSOCIATED CONTENT}

\section{S Supporting Information}

The Supporting Information is available free of charge on the ACS Publications website at DOI: 10.1021/jacs.9b01394.

Details of the chemicals used, NCs synthesis; ligand exchange reactions; pellet fabrication, materials characterization (X-ray diffraction patterns, HRTEM, electrical and thermal characterization); stability measurement and additional notes (PDF)

\section{AUTHOR INFORMATION}

\section{Corresponding Authors}

*mibanez@ist.ac.at

*mvkovalenko@ethz.ch

\section{ORCID $\odot$}

Maria Ibáñez: 0000-0001-5013-2843

Yu Liu: 0000-0001-7313-6740

Oleksandr Dobrozhan: 0000-0001-9238-7596

Jordi Arbiol: 0000-0002-0695-1726

Andreu Cabot: 0000-0002-7533-3251

Maksym V. Kovalenko: 0000-0002-6396-8938

Notes

The authors declare no competing financial interest.

\section{ACKNOWLEDGMENTS}

This work was financially supported by the European Union (EU) via FP7 ERC Starting Grant 2012 (Project NANOSOLID, GA No. 306733). M.I. was supported by IST Austria, and by ETH Zurich via ETH career seed grant (SEED-18 16-2). Y.L. acknowledges funding from the European Union's Horizon 2020 research and innovation programme under the Marie Sklodowska-Curie grant agreement No. 754411. IREC acknowledges funding from Generalitat de Catalunya (2014SGR1638). J.A. acknowledge funding from Generalitat de Catalunya 2017 SGR 327 and the Spanish MINECO coordinated projects between IREC and ICN2 ENE2017-85087-C3. ICN2 acknowledges support from the Severo Ochoa Program (MINECO, Grant SEV-2017-0706) and is funded by the CERCA Programme/ Generalitat de Catalunya.

\section{REFERENCES}

(1) Snyder, G. J.; Toberer, E. S. Complex Thermoelectric Materials. Nat. Mater. 2008, 7 (2), 105-114.

(2) Pei, Y.; Wang, H.; Snyder, G. J. Band Engineering of Thermoelectric Materials. Adv. Mater. 2012, 24 (46), 6125-6135.

(3) Tan, G.; Zhao, L.-D.; Kanatzidis, M. G. Rationally Designing HighPerformance Bulk Thermoelectric Materials. Chem. Rev. 2016, 116 (19), 12123-12149.

(4) Ortega, S.; Ibáñez, M.; Liu, Y.; Zhang, Y.; Kovalenko, M. V.; Cadavid, D.; Cabot, A. Bottom-Up Engineering of Thermoelectric Nanomaterials and Devices from Solution-Processed Nanoparticle Building Blocks. Chem. Soc. Rev. 2017, 46, 3510-3528.

(5) Ibáñez, M.; Luo, Z.; Genc, A.; Piveteau, L.; Ortega, S.; Cadavid, D.; Dobrozhan, O.; Liu, Y.; Nachtegaal, M.; Zebarjadi, M.; Arbiol, J.; Kovalenko, M. V.; Cabot, A. High-Performance Thermoelectric Nanocomposites from Nanocrystal Building Blocks. Nat. Commun. 2016, 7, 10766.

(6) Liu, Y.; Garcia, G.; Ortega, S.; Cadavid, D.; Palacios, P.; Lu, J.; Ibáñez, M.; Xi, L.; De Roo, J.; Lopez, A. M.; Marti-Sanchez, S.; Cabezas, I.; Mata, M. d. 1.; Luo, Z.; Dun, C.; Dobrozhan, O.; Carroll, D. L.; Zhang, W.; Martins, J.; Kovalenko, M. V.; Arbiol, J.; Noriega, G.; Song, J.; Wahnon, P.; Cabot, A. Solution-Based Synthesis and Processing of Snand Bi-doped $\mathrm{Cu}_{3} \mathrm{SbSe}_{4}$ Nanocrystals, Nanomaterials and Ring-Shaped Thermoelectric Generators. J. Mater. Chem. A 2017, 5 (6), 2592-2602.

(7) Xu, J.; Li, H.; Du, B.; Tang, X.; Zhang, Q.; Uher, C. High Thermoelectric Figure of Merit and Nanostructuring in Bulk AgSbTe $2 . J$. Mater. Chem. 2010, 20 (29), 6138-6143.

(8) Xiao, C.; Xu, J.; Cao, B.; Li, K.; Kong, M.; Xie, Y. Solid-Solutioned Homojunction Nanoplates with Disordered Lattice: A Promising Approach toward "Phonon Glass Electron Crystal" Thermoelectric Materials. J. Am. Chem. Soc. 2012, 134 (18), 7971-7977.

(9) Xu, B.; Agne, M. T.; Feng, T.; Chasapis, T. C.; Ruan, X.; Zhou, Y.; Zheng, H.; Bahk, J.-H.; Kanatzidis, M. G.; Snyder, G. J.; Wu, Y. Nanocomposites from Solution-Synthesized PbTe-BiSbTe Nanoheterostructure with Unity Figure of Merit at Low-Medium Temperatures (500-600 K). Adv. Mater. 2017, 29 (10), 1605140.

(10) Liu, Y.; Zhang, Y.; Lim, K. H.; Ibáñez, M.; Ortega, S.; Li, M.; David, J.; Martí-Sánchez, S.; Ng, K. M.; Arbiol, J.; Kovalenko, M. V.; Cadavid, D.; Cabot, A. High Thermoelectric Performance in Crystallographically Textured $\mathrm{n}$-Type $\mathrm{Bi}_{2} \mathrm{Te}_{3-\mathrm{x}} \mathrm{Se}_{\mathrm{x}}$ Produced from Asymmetric Colloidal Nanocrystals. ACS Nano 2018, 12 (7), 7174-7184.

(11) Liu, Y.; Zhang, Y.; Ortega, S.; Ibáñez, M.; Lim, K. H.; GrauCarbonell, A.; Martí-Sánchez, S.; Ng, K. M.; Arbiol, J.; Kovalenko, M. V.; Cadavid, D.; Cabot, A. Crystallographically Textured Nanomaterials Produced from the Liquid Phase Sintering of $\mathrm{Bi}_{x} \mathrm{Sb}_{2-\mathrm{x}} \mathrm{Te}_{3}$ Nanocrystal Building Blocks. Nano Lett. 2018, 18 (4), 2557-2563.

(12) De Roo, J.; De Keukeleere, K.; Hens, Z.; Van Driessche, I. From Ligands to Binding Motifs and Beyond; the Enhanced Versatility of Nanocrystal Surfaces. Dalton Trans 2016, 45 (34), 13277-13283.

(13) Ibáñez, M.; Hasler, R.; Liu, Y.; Dobrozhan, O.; Nazarenko, O.; Cadavid, D.; Cabot, A.; Kovalenko, M. V. Tuning p-Type Transport in Bottom-Up-Engineered Nanocrystalline $\mathrm{Pb}$ Chalcogenides Using Alkali Metal Chalcogenides as Capping Ligands. Chem. Mater. 2017, 29 (17), 7093-7097.

(14) Ibáñez, M.; Korkosz, R. J.; Luo, Z.; Riba, P.; Cadavid, D.; Ortega, S.; Cabot, A.; Kanatzidis, M. G. Electron Doping in Bottom-Up Engineered Thermoelectric Nanomaterials through HCl-Mediated Ligand Displacement. J. Am. Chem. Soc. 2015, 137 (12), 4046-4049. 
(15) Zhang, H.; Son, J. S.; Dolzhnikov, D. S.; Filatov, A. S.; Hazarika, A.; Wang, Y.; Hudson, M. H.; Sun, C.-J.; Chattopadhyay, S.; Talapin, D. V. Soluble Lead and Bismuth Chalcogenidometallates: Versatile Solders for Thermoelectric Materials. Chem. Mater. 2017, 29 (15), 6396-6404.

(16) Son, J. S.; Zhang, H.; Jang, J.; Poudel, B.; Waring, A.; Nally, L.; Talapin, D. V. All-Inorganic Nanocrystals as a Glue for BiSbTe Grains: Design of Interfaces in Mesostructured Thermoelectric Materials. Angew. Chem. 2014, 126 (29), 7596-7600.

(17) Zhang, X.; Zhou, Y.; Pei, Y.; Chen, Y.; Yuan, B.; Zhang, S.; Deng, Y.; Gong, S.; He, J.; Zhao, L.-D. Enhancing thermoelectric performance of SnTe via nanostructuring particle size. J. Alloys Compd. 2017, 709, 575-580.

(18) Li, Z.; Chen, Y.; Li, J.-F.; Chen, H.; Wang, L.; Zheng, S.; Lu, G. Systhesizing SnTe nanocrystals leading to thermoelectric performance enhancement via an ultra-fast microwave hydrothermal method. Nano Energy 2016, 28, 78-86.

(19) Han, G.; Zhang, R.; Popuri, S.; Greer, H.; Reece, M.; Bos, J.-W.; Zhou, W.; Knox, A.; Gregory, D. Large-Scale Surfactant-Free Synthesis of p-Type SnTe Nanoparticles for Thermoelectric Applications. Materials 2017, 10 (3), 233.

(20) Tan, G.; Shi, F.; Hao, S.; Chi, H.; Zhao, L.-D.; Uher, C.; Wolverton, C.; Dravid, V. P.; Kanatzidis, M. G. Codoping in SnTe: Enhancement of Thermoelectric Performance through Synergy of Resonance Levels and Band Convergence. J. Am. Chem. Soc. 2015, 137 (15), 5100-5112.

(21) Fafarman, A. T.; Koh, W.-k.; Diroll, B. T.; Kim, D. K.; Ko, D.-K.; Oh, S. J.; Ye, X.; Doan-Nguyen, V.; Crump, M. R.; Reifsnyder, D. C.; Murray, C. B.; Kagan, C. R. Thiocyanate-Capped Nanocrystal Colloids: Vibrational Reporter of Surface Chemistry and Solution-Based Route to Enhanced Coupling in Nanocrystal Solids. J. Am. Chem. Soc. 2011, 133 (39), 15753-15761.

(22) Koh, W.-k.; Saudari, S. R.; Fafarman, A. T.; Kagan, C. R.; Murray, C. B. Thiocyanate-Capped PbS Nanocubes: Ambipolar Transport Enables Quantum Dot Based Circuits on a Flexible Substrate. Nano Lett. 2011, 11 (11), 4764-4767.

(23) Joffé, A. F. Heat Transfer in Semiconductors. Can. J. Phys. 1956, 34 (12A), 1342-1355.

(24) Kafalas, J. A.; Brebrick, R. F.; Strauss, A. J. Evidence that $\mathrm{SnTe}$ is a Semiconductor. Appl. Phys. Lett. 1964, 4 (5), 93-94.

(25) Littlewood, P. B.; Mihaila, B.; Schulze, R. K.; Safarik, D. J.; Gubernatis, J. E.; Bostwick, A.; Rotenberg, E.; Opeil, C. P.; Durakiewicz, T.; Smith, J. L.; Lashley, J. C. Band Structure of SnTe Studied by Photoemission Spectroscopy. Phys. Rev. Lett. 2010, 105 (8), 086404.

(26) Brebrick, R. F.; Strauss, A. J. Anomalous Thermoelectric Power as Evidence for Two-Valence Bands in SnTe. Phys. Rev. 1963, 131 (1), 104-110.

(27) Brebrick, R. F. Deviations from stoichiometry and electrical properties in SnTe. J. Phys. Chem. Solids 1963, 24 (1), 27-36.

(28) Zhou, M.; Snyder, G. J.; Li, L.; Zhao, L.-D. Lead-free tin chalcogenide thermoelectric materials. Inorg. Chem. Front. 2016, 3 (11), 1449-1463.

(29) Zhou, M.; Gibbs, Z. M.; Wang, H.; Han, Y.; Xin, C.; Li, L.; Snyder, G. J. Optimization of thermoelectric efficiency in SnTe: the case for the light band. Phys. Chem. Chem. Phys. 2014, 16 (38), 20741-20748.

(30) Zhao, L.-D.; Zhang, X.; Wu, H.; Tan, G.; Pei, Y.; Xiao, Y.; Chang, C.; Wu, D.; Chi, H.; Zheng, L.; Gong, S.; Uher, C.; He, J.; Kanatzidis, M. G. Enhanced Thermoelectric Properties in the Counter-Doped SnTe System with Strained Endotaxial SrTe. J. Am. Chem. Soc. 2016, 138 (7), 2366-2373.

(31) Tan, G.; Zhao, L.-D.; Shi, F.; Doak, J. W.; Lo, S.-H.; Sun, H.; Wolverton, C.; Dravid, V. P.; Uher, C.; Kanatzidis, M. G. High Thermoelectric Performance of p-Type SnTe via a Synergistic Band Engineering and Nanostructuring Approach. J. Am. Chem. Soc. 2014, 136 (19), 7006-7017.

(32) Zhang, X.; Wang, D.; Wu, H.; Yin, M.; Pei, Y.; Gong, S.; Huang, L.; Pennycook, S. J.; He, J.; Zhao, L.-D. Simultaneously Enhancing the Power Factor and Reducing the Thermal Conductivity of SnTe via Introducing its Analogues. Energy Environ. Sci. 2017, 10 (11), 24202431.
(33) He, J.; Xu, J.; Liu, G.-Q.; Shao, H.; Tan, X.; Liu, Z.; Xu, J.; Jiang, H.; Jiang, J. Enhanced thermopower in rock-salt SnTe-CdTe from band convergence. RSC Adv. 2016, 6 (38), 32189-32192.

(34) Tan, X. J.; Shao, H. Z.; He, J.; Liu, G. Q.; Xu, J. T.; Jiang, J.; Jiang, H.C. Band engineering and improved thermoelectric performance in Mdoped $\mathrm{SnTe}(\mathrm{M}=\mathrm{Mg}, \mathrm{Mn}, \mathrm{Cd}$, and Hg). Phys. Chem. Chem. Phys. 2016, 18 (10), 7141-7147.

(35) Dong, X.; Yu, H.; Li, W.; Pei, Y.; Chen, Y. First-Principles Study on Band Structures and Electrical Transports of Doped-SnTe. J. Materiomics 2016, 2 (2), 158-164.

(36) Tan, G.; Shi, F.; Doak, J. W.; Sun, H.; Zhao, L.-D.; Wang, P.; Uher, C.; Wolverton, C.; Dravid, V. P.; Kanatzidis, M. G. Extraordinary role of $\mathrm{Hg}$ in enhancing the thermoelectric performance of p-type SnTe. Energy Environ. Sci. 2015, 8 (1), 267-277.

(37) Banik, A.; Shenoy, U. S.; Anand, S.; Waghmare, U. V.; Biswas, K. $\mathrm{Mg}$ Alloying in SnTe Facilitates Valence Band Convergence and Optimizes Thermoelectric Properties. Chem. Mater. 2015, 27 (2), 581587.

(38) Al Rahal Al Orabi, R.; Mecholsky, N. A.; Hwang, J.; Kim, W.; Rhyee, J.-S.; Wee, D.; Fornari, M. Band Degeneracy, Low Thermal Conductivity, and High Thermoelectric Figure of Merit in $\mathrm{SnTe}-\mathrm{CaTe}$ Alloys. Chem. Mater. 2016, 28 (1), 376-384.

(39) Roychowdhury, S.; Shenoy, U. S.; Waghmare, U. V.; Biswas, K. An enhanced Seebeck coefficient and high thermoelectric performance in $\mathrm{p}$ type In and $\mathrm{Mg}$ co-doped Sn1-xPbxTe via the co-adjuvant effect of the resonance level and heavy hole valence band. J. Mater. Chem. C 2017, 5, 5737.

(40) Wu, H.; Chang, C.; Feng, D.; Xiao, Y.; Zhang, X.; Pei, Y.; Zheng, L.; Wu, D.; Gong, S.; Chen, Y.; He, J.; Kanatzidis, M. G.; Zhao, L.-D. Synergistically optimized electrical and thermal transport properties of SnTe via alloying high-solubility MnTe. Energy Environ. Sci. 2015, 8 (11), 3298-3312.

(41) Tan, G.; Shi, F.; Sun, H.; Zhao, L.-D.; Uher, C.; Dravid, V. P.; Kanatzidis, M. G. SnTe-AgBiTe2 as an efficient thermoelectric material with low thermal conductivity. J. Mater. Chem. A 2014, 2 (48), 2084920854.

(42) Banik, A.; Shenoy, U. S.; Saha, S.; Waghmare, U. V.; Biswas, K. High Power Factor and Enhanced Thermoelectric Performance of SnTe-AgInTe2: Synergistic Effect of Resonance Level and Valence Band Convergence. J. Am. Chem. Soc. 2016, 138 (39), 13068-13075.

(43) Tan, G.; Zeier, W. G.; Shi, F.; Wang, P.; Snyder, G.J.; Dravid, V. P.; Kanatzidis, M. G. High Thermoelectric Performance SnTe-In2Te3 Solid Solutions Enabled by Resonant Levels and Strong Vacancy Phonon Scattering. Chem. Mater. 2015, 27 (22), 7801-7811.

(44) Al Rahal Al Orabi, R.; Hwang, J.; Lin, C.-C.; Gautier, R.; Fontaine, B.; Kim, W.; Rhyee, J.-S.; Wee, D.; Fornari, M. Ultralow Lattice Thermal Conductivity and Enhanced Thermoelectric Performance in SnTe:Ga Materials. Chem. Mater. 2017, 29 (2), 612-620.

(45) McCarthy, C. L.; Brutchey, R. L. Solution Processing of Chalcogenide Materials Using Thiol-Amine "Alkahest" Solvent Systems. Chem. Commun. 2017, 53 (36), 4888-4902.

(46) Dolzhnikov, D. S.; Zhang, H.; Jang, J.; Son, J. S.; Panthani, M. G.; Shibata, T.; Chattopadhyay, S.; Talapin, D. V. Composition-matched molecular "solders" for semiconductors. Science 2015, 347 (6220), 425.

(47) Hudson, M. H.; Dolzhnikov, D. S.; Filatov, A. S.; Janke, E. M.; Jang, J.; Lee, B.; Sun, C.; Talapin, D. V. New Forms of CdSe: Molecular Wires, Gels, and Ordered Mesoporous Assemblies. J. Am. Chem. Soc. 2017, 139 (9), 3368-3377. 